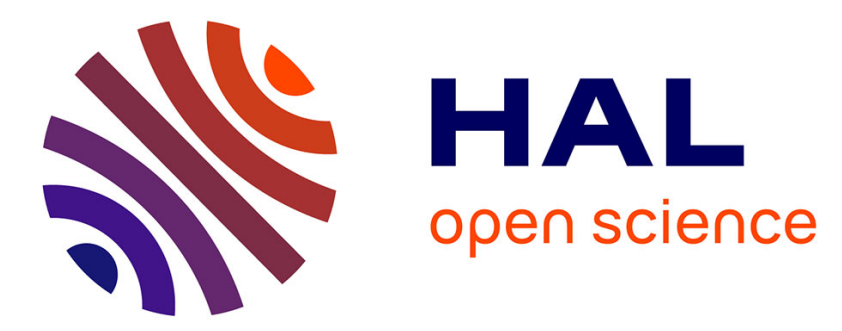

\title{
Modelling conjugation with stochastic differential equations
}

\author{
K.R. Philipsen, L.E. Christiansen, H. Hasman, H. Madsen
}

\section{To cite this version:}

K.R. Philipsen, L.E. Christiansen, H. Hasman, H. Madsen. Modelling conjugation with stochastic differential equations. Journal of Theoretical Biology, 2010, 263 (1), pp.134. 10.1016/j.jtbi.2009.11.011 . hal-00564469

\section{HAL Id: hal-00564469 \\ https://hal.science/hal-00564469}

Submitted on 9 Feb 2011

HAL is a multi-disciplinary open access archive for the deposit and dissemination of scientific research documents, whether they are published or not. The documents may come from teaching and research institutions in France or abroad, or from public or private research centers.
L'archive ouverte pluridisciplinaire HAL, est destinée au dépôt et à la diffusion de documents scientifiques de niveau recherche, publiés ou non, émanant des établissements d'enseignement et de recherche français ou étrangers, des laboratoires publics ou privés. 


\section{Author's Accepted Manuscript}

Modelling conjugation with stochastic differential equations

K.R. Philipsen, L.E. Christiansen, H. Hasman, H. Madsen

PII: S0022-5193(09)00553-0

DOI:

Reference: doi:10.1016/j.jtbi.2009.11.011 YJTBI 5776

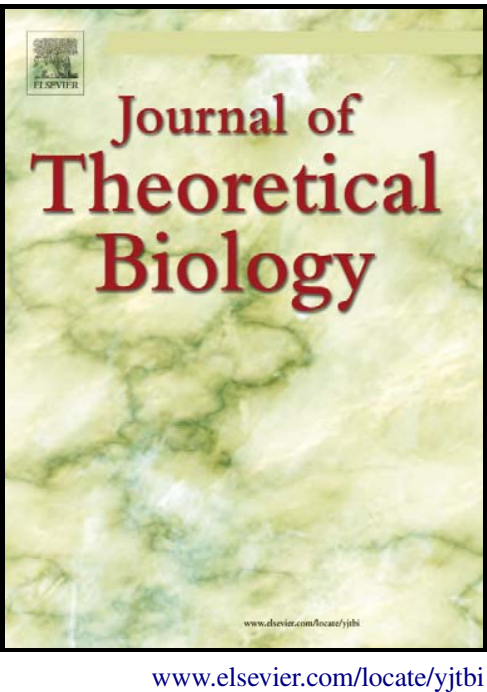

To appear in: Journal of Theoretical Biology

Received date: 18 June 2009

Revised date: $\quad 21$ September 2009

Accepted date: 17 November 2009

Cite this article as: K.R. Philipsen, L.E. Christiansen, H. Hasman and H. Madsen, Modelling conjugation with stochastic differential equations, Journal of Theoretical Biology, doi:10.1016/j.jtbi.2009.11.011

This is a PDF file of an unedited manuscript that has been accepted for publication. As a service to our customers we are providing this early version of the manuscript. The manuscript will undergo copyediting, typesetting, and review of the resulting galley proof before it is published in its final citable form. Please note that during the production process errors may be discovered which could affect the content, and all legal disclaimers that apply to the journal pertain. 


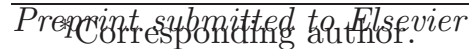

Email address: krp@imm.dtu.dk (K. R. Philipsen)

URL: http://www.imm.dtu.dk/ $\operatorname{krp}$ (K. R. Philipsen) 
Key words: SDE, horizontal gene transfer, resistance, CTSM, E. faecium, mathematical modelling, Hidden Markov Model, likelihood-ratio test, AIC

\section{Introduction}

Development and spread of antimicrobial resistance in bacterial populations is of increasing concern, as it can lead to major difficulties for the treatment of diseases. A first step in the direction of solving this problem is to gain a better understanding of the spread of resistance, and here conjugation plays a major role. Conjugation is one of several mechanisms of horizontal gene transfer by which plasmids coding for e.g. antimicrobial resistance can be transferred between bacteria. The use of mathematical modelling to describe the dynamics of plasmid spread and persistence was first introduced by Levin et al. (1979) and since then many studies have been made to improve the model framework and parameter estimation and to incorporate more accurate expressions for the plasmid dynamics (Slater et al., 2008). Most studies have used ordinary differential equations, ODE (Levin et al., 1979; Freter et al., 1983; Clewlow et al., 1990; MacDonald et al., 1992; Willms et al., 2006), or ordinary difference equations (Knudsen et al., 1988; Sudarshana and Knudsen, 2006) as the modelling framework. This approach can be discussed since a part of the complexity of conjugation, e.g. dependence on the surrounding environment, is not included in these models. A way of overcoming this problem is to use a stochastic modelling approach where the randomness accounts for those processes not included in 
the model. Some efforts have been made to include randomness in plasmid models (Joyce et al., 2005; De Gelder et al., 2007; Ponciano et al., 2007). Common for these studies is that they use discrete-time models, which is a good approximation at low bacteria densities, but not at higher densities where growth and conjugation is a continuous process. Joyce et al. (2005) gives a first and second order moment representation of their model which requires a derivation of the means and variances. Thus, it is not easy to transfer the method to other models. In the study by Ponciano et al. (2007) one of the model parameters is implemented as a normal distributed random variable. This paper presents a generic modelling framework first described by Kristensen et al. (2004a), which is based on stochastic differential equations, SDE. The SDE is connected to data through a state-space formulation consisting of continuous-time state equations (the SDE) and discrete-time observation equations. The applied state-space approach is in fact a continuous time Hidden Markoy Model. The state-space formulation opens up for strong statistical tools for estimating model parameters and for inference concerning the best model (Kristensen et al., 2004a). State-space modelling has also been used in previous plasmid studies (De Gelder et al., 2004, 2007; Ponciano et al., 2007), but our approach differs by enabling continuous-time state equations in combination with discrete-time observations. The statespace model enables a simultaneous estimation of the growth and conjugation parameters, whereby the bacterial growth is accounted for when estimating the conjugation rate. This is an improvement from previous studies, where 
the donor and recipient concentrations were introduced as a mean value of two measurements taken over time (Knudsen et al., 1988; Sudarshana and Knudsen, 2006) or the experiment was constructed such that bacterial growth could be neglected (MacDonald et al., 1992).

In this article the SDE based state-space modelling framework is applied to analyze data from an in vitro experiment for conjugation between two Enterococcus faecium species growing in a batch exhaustable media. This experiment was made in order to study the transferability of vancomycin resistance in E. faecium. To describe the experimental system a conjugation model of bacteria growing in a broth exhaustible media is introduced, which is an expansion of previous models. Several authors (Levin and Stewart, 1980; Freter et al., 1983; Knudsen et al., 1988; Clewlow et al., 1990; Top et al., 1992; Willms et al., 2006; Sudarshana and Knudsen, 2006) have modelled conjugation events with the mass action model proposed by Levin et al. (1979). The mass action model states that the appearance of transconjugants is proportional to the product of the donor and recipient concentrations. We will introduce a new expression for the conjugation rate in an exhaustible media for which the proportionality constant of the mass action model is substrate dependent. An inference study is made to reduce the SDE model to its minimum form. A further extension of the model is made to treat adequately a methodological problem: the finding that conjugation continues on the agar plates selecting for transconjugants. In that case the observed transconjugants are a combination of transconjugants stemming from the 
conjugation process in the flask and conjugation occurring on the selective plates. The models with and without conjugation on the selective plates are compared in order to examine which model best describes data.

\section{Experimental methods}

A conjugation experiment was made in order to study the transferability of vancomycin resistance in Enterococcus faecium. As recipient the E. faecium reference strain BM4105RF was used. This strain is resistant to rifampicin (MIC $>25 \mu \mathrm{g} / \mathrm{ml}$ ) and fusidic acid $(\mathrm{MIC}>25 \mu \mathrm{g} / \mathrm{ml})$ due to chromosomally located mutations. As donor, the E. faecium A17sv1 (Hasman and Aarestrup, 2002) was used. This strain is resistant to erythromycin (MIC $>16 \mu \mathrm{g} / \mathrm{ml}$ ) and vancomycin (MIC $>32 \mu \mathrm{g} / \mathrm{ml}$ ) due to the presence of the $\operatorname{erm}(\mathrm{B})$ gene and $\operatorname{Tn} 1546$ transposon (carrying the vanA-gene cluster), respectively, located on a conjugative plasmid.

The conjugation experiment was performed in liquid Brain-Heart Infusion (BHI) media (Oxoid). Bacterial counting was performed on BHI agar plates supplemented with the following antibiotics when appropriate: rifampicin 25 $\mu \mathrm{g} / \mathrm{ml}$, fusidic acid $(25 \mu \mathrm{g} / \mathrm{ml})$, erythromycin $(16 \mu \mathrm{g} / \mathrm{ml})$ and vancomycin $(32 \mu \mathrm{g} / \mathrm{ml})$.

\subsection{Conjugation experiment}

Over night blood agar cultures of the two strains grown at $37^{\circ} \mathrm{C}$ were inoculated in BHI media supplemented with the appropriate antibiotics as 
described above. From these, $100 \mu \mathrm{l}$ of each culture was transferred to fresh tubes containing $10 \mathrm{ml}$ preheated BHI media without antibiotics supplementation. When these cultures reached late exponential growth $\left(\mathrm{OD}_{500}\right.$ of 0.3-0.5), the number of cells in each culture was adjusted to the same amount and $1.5 \mathrm{ml}$ of each was transferred to $100 \mathrm{ml}$ preheated $\left(37^{\circ} \mathrm{C}\right) \mathrm{BHI}$ media in an $250 \mathrm{ml}$ Erlenmeyer flask. The flask was placed in a shaking incubator $(125 \mathrm{rpm})$ at $37^{\circ} \mathrm{C}$. Four $1 \mathrm{ml}$ samples were taken immediately after the cells were added $(\mathrm{t}=0)$ as well as 1.50, 2.80, 3.00, 3.40, 3.80, 4.00, 4.40, 4.80, 5.00, $5.50,6.00,6.30$ and 7.00 hours after the cells were mixed. One of the four $1 \mathrm{ml}$ samples was used to measure the $\mathrm{OD}_{500}$ of the culture. The remaining three samples were diluted ten-fold until $10^{-7}$. From here, $100 \mu$ l of each dilution (where appropriate) was plated onto sets of plates containing BHI agar supplemented with either of the following: 1) Recipient plates containing $25 \mu \mathrm{g} / \mathrm{ml}$ rifampicin $+25 \mu \mathrm{g} / \mathrm{ml}$ fusidic acid, 2) Donor plates containing $16 \mu \mathrm{g} / \mathrm{ml}$ erythromycin $+32 \mu \mathrm{g} / \mathrm{ml}$ vancomycin, and 3) transconjugant plates containing $25 \mu \mathrm{g} / \mathrm{ml}$ rifampicin $+25 \mu \mathrm{g} / \mathrm{ml}$ fusidic acid $+16 \mu \mathrm{g} / \mathrm{ml}$ erythromycin $+32 \mu \mathrm{g} / \mathrm{ml}$ vancomycin. All plates were incubated 48 hours and the colony forming units, CFU, were counted. From these numbers the bacterial concentration in the flask was calculated.

\subsection{Calculating bacterial concentration}

The concentrations of the different bacteria populations in the flask can be determined from the plate count by a generalized linear model approach, 
assuming that the $\mathrm{CFU}$ count for each plate is Poisson distributed with an offset corresponding to the given dilution. If, for any given observation time $t_{k}, N_{j k}$ is the count number $j$ with dilution $n_{j}$, then the expected CFU count $E\left(N_{j k}\right)$ can be modelled with the generalized linear model

$$
\begin{aligned}
& E\left(N_{j k}\right)=\lambda_{j k}=n_{j} \exp \left(\beta_{k}\right), N_{j k} \sim \operatorname{Poisson}\left(\lambda_{j k}\right) \\
& \log \left(\lambda_{j k}\right)=\log \left(n_{j}\right)+\beta_{k} .
\end{aligned}
$$

Fitting the model to data gives an estimate for the coefficient $\beta_{k}$ and hereby an estimate of the bacterial concentration $Y_{k}=\exp \left(\beta_{k}\right)$ in the flask at time, $t_{k}$. The variance $\sigma_{Y, k}^{2}$ of the estimated concentration at time $t_{k}$ can be determined from the variance of $\beta$ as

$$
\sigma_{Y, k}^{2}=\operatorname{Var}\left[\exp \left(\beta_{k}\right)\right]=\left(\exp \left(\beta_{k}\right) \operatorname{Var}[\beta]\right)^{2} .
$$

The concentrations $Y_{k}$ calculated in this way are the observations to be used for the modelling procedure described in the reminder of this article. The command glmfit in Matlab is used for fitting the generalized linear model (see Appendix A).

\section{Model formulation}

The modelling framework used in this study is a continuous-discrete time state-space model consisting of a continuous time state equation (the SDE) and a discrete time observation equation. The model has the general form 
(Kristensen et al., 2004b)

$$
\begin{aligned}
d \boldsymbol{X}_{t} & =\boldsymbol{f}\left(\boldsymbol{X}_{t}, \boldsymbol{u}_{t}, t, \boldsymbol{\theta}\right) d t+\boldsymbol{\sigma}\left(\boldsymbol{u}_{t}, t, \boldsymbol{\theta}\right) d \boldsymbol{\omega}_{t} \\
\boldsymbol{Y}_{k} & =\boldsymbol{h}\left(\boldsymbol{X}_{k}, \boldsymbol{u}_{k}, t_{k}, \boldsymbol{\theta}\right)+\boldsymbol{e}_{k}
\end{aligned}
$$

where (4) is the SDE and (5) is the observation equation. $\boldsymbol{X}_{t}$ is a $n$ dimensional vector of state variables and $\boldsymbol{Y}_{k}$ is a $l$-dimensional vector of observations. The observations are obtained at discrete times $t_{k}$ with the observation noise being $\boldsymbol{e}_{k} \in N\left(\mathbf{0}, \boldsymbol{\Sigma}_{\mathbf{k}}\right)$. $\boldsymbol{\theta}$ is a vector of unknown parameters and $\boldsymbol{u}$ is a vector of input variables, i.e. variables which can be observed and have an influence on the system dynamics. The functions $\boldsymbol{f}$ and $\boldsymbol{h}$ can be linear as well as nonlinear functions. $\left\{\boldsymbol{\omega}_{t}\right\}$ is a standard Wiener process representing sources of noise in the system. The first part on the right-hand side of the SDE (4) is called the drift term and the second part is called the diffusion term. The stochastic state-space model (4)-(5) has several advantages compared to deterministic models. For instance, the state-space approach separates the residual noise into system noise and observation noise, where the system noise is used to e.g. compensate for those biological processes not explicitly described by the model. The deterministic model often leads to autocorrelated residuals, which is not a problem with the SDE. Using the SDE based model also paves the way for strong statistical tools to estimate model parameters and make inferences. 


(
gant concentrations. The flow diagram shows how recipients can be turned

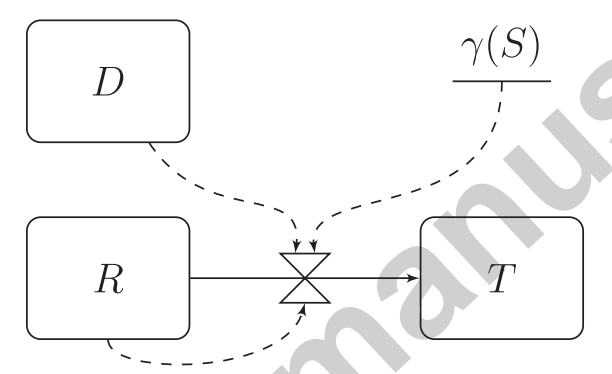

Figure 1: Flow diagram showing the structure of the conjugation models. The donor $D$ can by encounter of a recipient $R$ transfer a plasmid coding for resistance to the recipient. The recipient hereby is turned into a transconjugant $T$, which expresses the same antibiotic resistances as both the donor and the recipient.

175

\subsection{The drift term - Model for conjugation}

In this study we apply the state-space modelling framework on a model for conjugation. The first step of the modelling procedure is to formulate the drift part of the SDE based on microbiological knowledge of the system. A sketch of the conjugation dynamics is given by the flow diagram in Figure 1, where $D$ is the donor, $R$ the recipient, and $T$ the transconju-

into transconjugants with a rate dependent on the concentration of donor and recipient. In earlier studies (MacDonald et al., 1992; Knudsen et al., 1988; Sudarshana and Knudsen, 2006) the substrate concentration and/or donor and recipient concentrations were kept constant in the experiments and were thus held constant in the model. In order to better approximate in vivo situations, these constraints were not applied to our E. faecium conjugation experiment, and thus all three populations as well as the substrate content must be included as state variables in the model. The drift term 
corresponding to the model shown in Figure 1 is

$$
\boldsymbol{f}\left(\boldsymbol{X}_{t}, \boldsymbol{\theta}\right)=\left[\begin{array}{c}
\mu_{D}(S) D \\
\mu_{R}(S) R-\gamma(S) D R \\
\mu_{T}(S) T+\gamma(S) D R \\
-\eta\left(\mu_{D}(S) D+\mu_{R}(S) R+\mu_{T}(S) T\right)
\end{array}\right]
$$

where $\mu_{i}(S)$ is the growth rate for the bacteria population $i(D, R$ or $T)$, and $\gamma(S)$ is the conjugation rate. $\boldsymbol{X}=[D, R, T, S]$ are the model state variables and $\boldsymbol{\theta}=\left[v_{D}, v_{R}, v_{T}, \kappa_{D}, \kappa_{R}, \kappa_{T}, \gamma_{\max }, \kappa_{c}, \eta\right]$ are the parameters for the model. The substrate, $S$, is simulated as a normalized variable with an initial value of 1 . The amount of substrate in the solution decreases as it is utilized for bacterial growth. The parameter $\eta$ is the amount of normalized substrate used for each cell division.

Bacterial growth continues until the substrate is exhausted, and the stationary state has been reached. The growth rate can be modelled with the well known Monod relation (Monod, 1949)

$$
\mu_{i}(S)=\frac{v_{i} S}{\kappa_{i}+S}, S \in[0,1]
$$

where $S$ is the substrate concentration, $v_{i}$ is the maximum growth rate and $\kappa_{i}$ is the substrate concentration when the growth rate is half of its maximum value. Both parameters are specific for a given bacteria population $i$.

The conjugation event depends on the probability of an encounter between 
donor and recipient. In the original mass action model the conjugation rate $\gamma$ was introduced as a constant parameter. Several authors (Knudsen et al., 1988; Andrup et al., 1998; Andrup and Andersen, 1999; Ponciano et al., 2007) have discussed this assumption. Levin et al. (1979) showed that the mass action model presents a good estimation of the transconjugant population during exponential growth and under chemostatic conditions, but the model fails to describe the occurrences of transconjugants during the lag phase and at the onset of stationary phase. MacDonald et al. (1992) suggested that the conjugation rate depends on the substrate content and stated that conjugation can not occur without the presence of nutrition. However, to our knowledge this substrate dependence has not before been implemented in a mathematical model for conjugation. We suggest a nonlinear substrate dependent expression to model the conjugation rate

$$
\gamma(S)=\frac{\gamma_{\max } S}{\kappa_{c}+S}, S \in[0,1]
$$

This expression is similar to the Michaelis-Menten equation for enzyme kinetics and to the Monod relation. This expression is chosen as it forces the conjugation rate to reach a maximum value $\gamma_{\max }$ when the substrate concentration is abundant, and it turns to zero as the substrate is depleted. Depending on the value of $\kappa_{c}$ the conjugation rate will decrease concurrent with or after the decrease in the bacterial growth rate.

Some assumptions are applied to keep the conjugation model simple. $(i)$ 
Transconjugants can function as donors transferring a plasmid to a recipient, but this is not described by the model. It is assumed reasonable to omit it for the E. faecium data set used in this study, as the concentration of transconjugants is very low compared to the donor concentration, and therefore does not contribute significantly to conjugation. (ii) The delay on 10-15 min that has been found (Andrup et al., 1998) between two conjugation events for the same donor is assumed to be insignificant. During the experiment there is always a large number of donors not involved in a conjugation event and thus ready to start the conjugation by encounter of a recipient. Therefore the delay is disregarded. (iii) It is assumed that the maximum growth rate of the transconjugants is either the same as the maximum growth rate for the recipient or smaller due to a fitness cost of the plasmid, i.e. $v_{T}=v_{R}(1-\alpha)$, where $\alpha \in[0,1[$. An inference study will be made to test if $\alpha=0$.

\subsection{The diffusion term}

Depending on the system which is modelled it can be adequate to implement additive system noise, i.e. noise independent on the state variables, or multiplicative system noise, i.e. where the noise depends on the state variables. The choice of the system noise depends on assumption about the system modelled. Tier and Floyd (1981) have described how different assumption of a biological process can lead to either demographic stochasticity (where the variance is proportional to the state variable) or environmental stochasticity (where the variance is proportional to the state variable 
squared). In our system the noise is implemented as multiplicative (environmental) noise, as the random fluctuations affect the whole population and not only the growth process. For instance the mass action model has shown to be good during exponential growth but not during lag-phase and stationary phase. Therefore multiplicative noise can be implemented to account for those processes not well described by the model. The method used for evaluating the likelihood function (Kristensen et al., 2004b) requires that the diffusion term is independent of the state variables. Therefore, instead of the state variables $D, R$ and $T$, the input vectors $\boldsymbol{u}_{D}, \boldsymbol{u}_{R}$ and $\boldsymbol{u}_{T}$, which contains observations for the three states, are inserted as a scaling for the standard deviation. The noise term is

$$
\boldsymbol{\sigma}_{c}^{m}=\left[\begin{array}{cccc}
\sigma_{D} \boldsymbol{u}_{D} & 0 & 0 & 0 \\
0 & \sigma_{R} \boldsymbol{u}_{R} & 0 & 0 \\
0 & 0 & \sigma_{T} \boldsymbol{u}_{T} & 0 \\
-\eta \sigma_{D} \boldsymbol{u}_{D} & -\eta \sigma_{R} \boldsymbol{u}_{R} & -\eta \sigma_{T} \boldsymbol{u}_{T} & \sigma_{S}
\end{array}\right],
$$

where an increment in donor, recipient or transconjugant concentration lower the substrate content in order to keep the mass balance, and $\sigma_{S}$ is introduced in order to ensure stability and is fixed to a small value.

An additive noise term $\boldsymbol{\sigma}_{c}^{a}$ is also introduced to compare with the multiplicative noise. This is done, as it is not sure that the data set is sufficient to make a good prediction of the system noise, and therefore a simplification of 
the noise structure can be an advantage. The additive noise term is

$$
\boldsymbol{\sigma}_{c}^{a}=\left[\begin{array}{cccc}
\sigma_{D} & 0 & 0 & 0 \\
0 & \sigma_{R} & 0 & 0 \\
0 & 0 & \sigma_{T} & 0 \\
-\eta \sigma_{D} & -\eta \sigma_{R} & -\eta \sigma_{T} & \sigma_{S}
\end{array}\right]
$$

\subsection{Observation equation}

The observation equation relates observations to the state variables. In that way the state variables do not need to be measured directly and it is not necessary to have observations related to all state variables. In this study three of the state variables (donor, recipient and transconjugant) are observed. The concentration for the donor, recipient and transconjugant has been calculated from the CFU count as described in Section 2.2. We will refer to this as the observed concentration. The observation equation, when only conjugation in the broth media is considered, is

$$
\left[\begin{array}{l}
Y_{1} \\
Y_{2} \\
Y_{3}
\end{array}\right]_{k}=\left[\begin{array}{l}
D \\
R \\
T
\end{array}\right]_{k}+\boldsymbol{e}_{k}, \boldsymbol{e}_{k} \in N\left(\mathbf{0}, \boldsymbol{\Sigma}_{\mathbf{k}}\right)
$$

The model with this observation equation is called the Broth model.

In addition to plasmid transfer in the broth, we have found that conjugation can occur on the transconjugant plates. This is a surprising result as antibi- 
otics on the agar plate are traditionally considered to stop the conjugation process. However we have performed several experiments (data not shown), which have revealed that conjugation does also occur on the transconjugant plates. As the experiment is made to measure conjugation in the broth media we wish to separate conjugation on the plate from that in the flask. The following set of ODE's is suggested to model conjugation on the plate for each observation time $k$

$$
\begin{aligned}
& \frac{d D_{p k}}{d t}=-\lambda D_{p k} \\
& \frac{d R_{p k}}{d t}=-\lambda R_{p k}-\gamma_{p} D_{p k} R_{p k} \\
& \frac{d T_{p k}}{d t}=\gamma_{p} D_{p k} R_{p k}
\end{aligned}
$$

The initial values for the concentration of donor, $D_{p k}$, and recipient, $R_{p k}$, on the plate correspond to the concentrations in the flask at the time for the observation. Due to antibiotics on the plate, the donors and recipients die with a death rate $\lambda$ and even though substrate is present in abundant amounts it is assumed that the donor and recipient bacteria can not grow. The recipients which receives a plasmid on the agar plate (and thus become a transconjugants) become resistant to all the antibiotics present and can therefore grow and form colonies on the plate. The plate conjugation rate $\gamma_{p}$ is assumed to be independent off the substrate content as the substrate will not be depleted before the conjugation process stops.

The plate conjugation is modelled by finding the analytical solution for the 
ODE and inserting the solution in the observation equation for transconjugants Eq. (11). As the number of recipients receiving a plasmid on the plate is small, the last term in Eq. (13) can be disregarded. The equation to be solved is thus

$$
\frac{d T_{p k}}{d t}=\gamma_{p} D_{p k}(t) R_{p k}(t)
$$

where

$$
\begin{aligned}
& D_{p k}(t)=D_{k 0} \exp (-\lambda t), \\
& R_{p k}(t)=R_{k 0} \exp (-\lambda t) .
\end{aligned}
$$

The solution is

$$
T_{p k}=\frac{\gamma_{p}}{2 \lambda} D_{k 0} R_{k 0}(1-\exp (-2 \lambda t))
$$

which for $t \rightarrow \infty$ is

$$
T_{p k}=\frac{\gamma_{p}}{2 \lambda} D_{k 0} R_{k 0}
$$


300 301

302

303 304

305

306

307

parameter $\gamma_{p}^{\prime}$. Inserting the result into Eq. (11) leads to the observation equation for the so-called Broth-plate model

$$
\left[\begin{array}{c}
Y_{1} \\
Y_{2} \\
Y_{3}
\end{array}\right]_{k}=\left[\begin{array}{c}
D \\
R \\
T+\frac{\gamma_{p}^{\prime}}{2} D R
\end{array}\right]_{k}+\boldsymbol{e}_{k}, \boldsymbol{e}_{k} \in N(\mathbf{0}, \boldsymbol{\Sigma}) .
$$

\subsection{Observation noise}

Three different covariance matrices for the observation equation are implemented and compared

Additive:

$$
\Sigma_{k}^{a}=\left[\begin{array}{ccc}
s_{1}^{2} & 0 & 0 \\
0 & s_{2}^{2} & 0 \\
0 & 0 & s_{3}^{2}
\end{array}\right]
$$

Proportional:

$$
\Sigma_{k}^{p}=\left[\begin{array}{ccc}
s_{4}^{2} \sigma_{D, k}^{2} & 0 & 0 \\
0 & s_{5}^{2} \sigma_{R, k}^{2} & 0 \\
0 & 0 & s_{6}^{2} \sigma_{T, k}^{2}
\end{array}\right]
$$

Additive+Proportional:

$$
\Sigma_{k}^{a, p}=\left[\begin{array}{ccc}
s_{1}^{2}+s_{4}^{2} \sigma_{D, k}^{2} & 0 & 0 \\
0 & s_{2}^{2}+s_{5}^{2} \sigma_{R, k}^{2} & 0 \\
0 & 0 & s_{3}^{2}+s_{6}^{2} \sigma_{T, k}^{2}
\end{array}\right]
$$


Hence, the observations $Y_{1}, Y_{2}$ and $Y_{3}$ are assumed to be uncorrelated in all cases. The variances $\sigma_{R, k}^{2}, \sigma_{R, k}^{2}$ and $\sigma_{T, k}^{2}$ are estimates from Eq. (3) at time $t_{k}$. The most simple noise form is the additive noise, but it is only reasonable, if the variance of the observations is independent of the observations. If this is not the case one alternative is to transform the data to stabilize the variance. In this article instead a proportional noise term is suggested, which include the estimated variance of the observations. Additionally a covariance matrix is considered with both additive and proportional noise.

\subsection{Statistical methods}

The modelling procedure consist of several steps of parameter estimations and goodness of fit statistics. First the Broth model and Broth-plate models are reduced separately, i.e. the likelihood function is optimized for different implementation of the system noise, observation noise and drift term. The best fit for the Broth and Broth-plate models are found applying goodness of fit statistics based on a ML approach. The inference study is made using a likelihood-ratio test and Akaike Information Criterion, AIC. After reducing the Broth and Broth-plate models they are compared, again using a likelihood-ratio test and AIC. Following the parameter estimation the models are simulated in Matlab, by implementing a numerical Euler method as described by Higham (2001). 


\subsubsection{Parameter estimation}

A ML estimation method is used to determine the SDE model parameters. The parameters are found as those maximizing the likelihood function

$$
\mathcal{L}\left(\boldsymbol{\theta} ; \mathcal{Y}_{N}\right)=\left(\prod_{k=1}^{N} p\left(\boldsymbol{Y}_{k} \mid \mathcal{Y}_{k-1}, \boldsymbol{\theta}\right)\right) p\left(\boldsymbol{Y}_{0} \mid \boldsymbol{\theta}\right)
$$

for the sequence of observations $\mathcal{Y}_{N}=\left[\boldsymbol{Y}_{N}, \boldsymbol{Y}_{N-1}, \ldots, \boldsymbol{Y}_{1}, \hat{\boldsymbol{Y}}_{0}\right]$. The conditional probability densities $p$ are approximated by gaussian densities motivated by the fact that the SDE (4) is driven by a Wiener process having gaussian increments, i.e.

where

$$
p\left(\boldsymbol{Y}_{k} \mid \mathcal{Y}_{k-1}, \boldsymbol{\theta}\right)=\frac{\exp \left(-\frac{1}{2} \boldsymbol{\epsilon}_{k}^{T}\left(\boldsymbol{\Sigma}_{k \mid k-1}^{y y}\right)^{-1} \boldsymbol{\epsilon}_{k}\right)}{\sqrt{\operatorname{det}\left(\boldsymbol{\Sigma}_{k \mid k-1}^{y y}\right)}(\sqrt{2 \pi})^{l}}
$$

$$
\begin{aligned}
\boldsymbol{\Sigma}_{k \mid k-1}^{y y} & =\operatorname{Var}\left[\boldsymbol{Y}_{k} \mid \mathcal{Y}_{k-1}, \boldsymbol{\theta}\right], \\
\boldsymbol{\epsilon}_{k} & =\boldsymbol{Y}_{k}-\hat{\boldsymbol{Y}}_{k \mid k-1}, \text { and } \\
\hat{\boldsymbol{Y}}_{k \mid k-1} & =\mathrm{E}\left[\boldsymbol{Y} \mid \mathcal{Y}_{k-1}, \boldsymbol{\theta}\right] .
\end{aligned}
$$

The conditional mean $\hat{\boldsymbol{Y}}_{k \mid k-1}$ and covariance $\boldsymbol{\Sigma}_{k \mid k-1}^{y y}$ in the likelihood function (24) and (25) can be estimated recursively by means of the Extended Kalman Filter (Kristensen et al., 2004b). In this study we use the software CTSM, from which the parameter estimation, correlation of the parameter estimates and the log-likelihood values are obtained. CTSM can be downloaded from the 
webpage: http://www2.imm.dtu.dk/ ctsm/, from where a user manual is also available. CTSM is easy to use as it has a built in graphical user interface. Recently an implementation of the method has also been made in R (Klim et al., 2009).

\subsubsection{Goodness of fit statistics}

Nested models are compared with a likelihood ratio test statistics given by

$$
-2 \log \Lambda=2\left(\ell(\boldsymbol{\theta})-\ell\left(\boldsymbol{\theta}_{0}\right)\right)
$$

where the test statistics $-2 \log \Lambda$ is asymptotically $\chi^{2}$ distributed with degrees of freedom equal to the difference in dimensions between the two models. $\ell(\boldsymbol{\theta})=\log \left(\mathcal{L}\left(\boldsymbol{\theta} ; \mathcal{Y}_{N}\right)\right)$ and $\ell\left(\boldsymbol{\theta}_{0}\right)=\log \left(\mathcal{L}\left(\boldsymbol{\theta}_{0} ; \mathcal{Y}_{N}\right)\right)$ are the log-likelihood values of the model and the submodel, respectively. The inference study is also performed with AIC, which is given by

$$
A I C=-2 \ell(\boldsymbol{\theta})+2 k,
$$

where $k$ is the number of parameters in the model and $\ell(\boldsymbol{\theta})$ is the $\log$ likelihood value of the model. When comparing models the preferred model is the one with the lowest AIC value. 

experimental results can be seen in Figure 2.
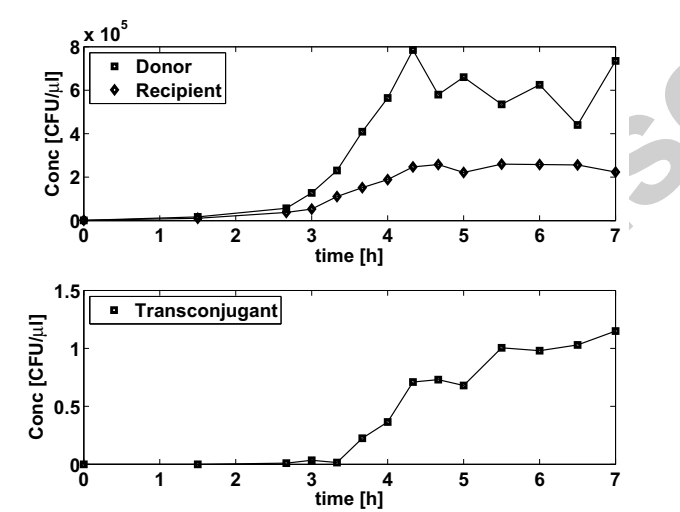

Figure 2: Data from the conjugation experiment with E. faecium growing in broth culture.

\section{Results and discussion}

The conjugation experiment continued for $7 \mathrm{~h}$, during which samples were taken from the broth mixture approximately every 20 min and plated on selective agar plates. The CFUs were counted, and the bacterial concentrations in the broth were determined by a generalized linear model approach. The

\subsection{Inference study}

Log-likelihood values for the Broth model and the Broth-plate model tested with respect to the state noise, the observation noise, and finally the drift term is summarized in Table 1 . When estimating the model parameters only biological plausible parameter intervals are considered. The multiplicative system noise term (9) is tested against additive system noise (10), and it is found that there is no significant difference. We therefore continue the study for both the Broth and Broth-plate model with additive system noise. 
Table 1: Log-likelihood values, p-values and AIC for different versions of the Broth model and Broth-plate model. The nested models listed below each other are tested with the likelihood-ratio test and the p-value for the comparison is listed in the line of the smallest of the two models tested. The proportional and additive observation noise structures are both tested against the proportional+additive noise term.

\begin{tabular}{lcccccc}
\hline & \multicolumn{3}{c}{ Broth model } & \multicolumn{3}{c}{ Broth-plate model } \\
& $\ell(\hat{\boldsymbol{\theta}})$ & $\mathrm{p}$-value & AIC & $\ell(\hat{\boldsymbol{\theta}})$ & $\mathrm{p}$-value & AIC \\
\hline System noise: & -299.04 & - & 638.08 & -298.05 & - & 634.09 \\
\hline Multiplicative & -299.19 & - & 634.38 & -298.05 & - & 634.09 \\
Additive & -299.19 & 0.9999 & 630.38 & -298.05 & 0.9999 & 630.09 \\
Additive $\left(\sigma=\sigma_{D}=\sigma_{R}=\sigma_{T}\right)$ & & & & & & \\
Observation noise: & & & & & & \\
Proportional+additive & -299.19 & - & 630.38 & -298.05 & - & 630.09 \\
Proportional & -300.52 & 0.4467 & 627.04 & -299.47 & 0.4162 & 626.94 \\
Additive & -312.67 & 0.0006 & 651.34 & -311.43 & 0.0007 & 650.86 \\
Drift term: & & & & & & \\
$\alpha=0$ & -300.52 & 0.9969 & 625.04 & -299.47 & 0.9695 & 624.94 \\
$\kappa_{T}=\kappa_{R}$ & -300.59 & 0.7014 & 623.19 & -299.53 & 0.7232 & 623.06 \\
\hline
\end{tabular}

It would be interesting to investigate the system noise structure with a ML estimation based on the particle filter (Ionides et al., 2006), for which the multiplicative noise can be implemented directly dependent on the state variable. We will leave this for a future study. The additive system noise is well modelled with $\sigma=\sigma_{D}=\sigma_{R}=\sigma_{T}$ for both the Broth and Broth-plate models.

Both the proportional observation noise and the additive observation noise can be tested against the proportional+additive observation noise using a likelihood-ratio test. The model with additive observation noise is seen to perform significantly worse than the proportional+additive noise model (pvalue $=0.0006$ and $\mathrm{p}$-value $=0.0007)$, whereas data is well modelled with 
proportional observation noise $(\mathrm{p}$-value $=0.4467$ and $\mathrm{p}$-value $=0.4162)$. All the observation noise covariance matrices can be compared using AIC, from which it is also found that data is best modelled with proportional observation noise. This result is as expected, as the variance of the observations increases for higher CFU counts, which is best captured by proportional noise.

With this noise structure the two hypothesis $\alpha=0$ and $\kappa_{T}=\kappa_{R}$ are tested. We fail to reject both hypothesis as the p-values are high (between 0.7014 and 0.9969).

The best description of data for each of the models is thus a model with additive system noise where the standard deviation is the same for $D, R$ and $T$. The observation noise should be modelled as proportional noise and growth for the transconjugants and recipients can be modelled with the same set of parameters.

The reduced Broth and Broth-plate models can be compared with the likelihoodratio test, as they are nested models with the Broth-plate model containing one additional parameter $\left(\gamma_{p}^{\prime}\right)$ compared to the Broth model. The test statistics for this test is 2.12 , which gives a p-value of 0.1451 . This is not significant on ordinary level and thus the smaller Broth model should be chosen. However, this conclusion is based on very few datapoints, and it might change if more data was available. 


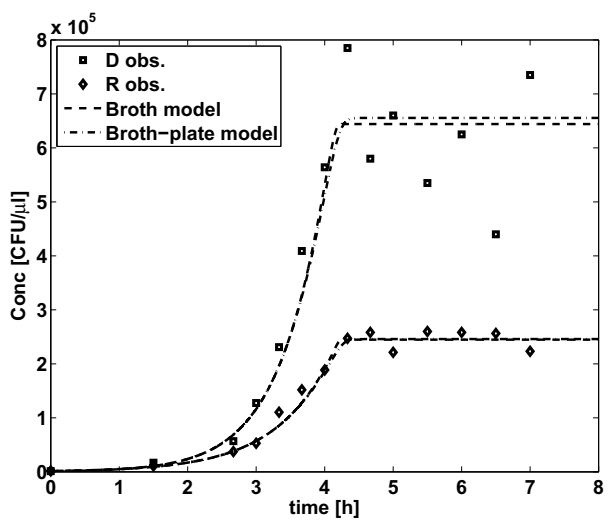

Figure 3: The donor and recipient concentration over time as simulated by the Broth and Broth-plate models plotted together with data values.

\subsection{Parameters}

The result of the ML parameter estimation for the reduced Broth and Broth-plate models is shown in Table 2, and the results of simulations with these parameters are plotted in Figure 3 and 4.

The conjugation rate is estimated to $2.008 \cdot 10^{-12}(\mathrm{CFU} / \mu \mathrm{l})^{-1} \mathrm{~h}^{-1}$ for the Broth-plate model and 4.913 $10^{-12}(\mathrm{CFU} / \mu \mathrm{l})^{-1} \mathrm{~h}^{-1}$ for the Broth model. Thus, the choice of model influence the estimate of the conjugation rate.

The value of $\kappa_{c}\left(10^{-16}-10^{-15}\right)$ is low compared to the $\kappa$ value for the growth of the bacteria $\left(10^{-3}-10^{-2}\right)$. This means that the mass action model $\gamma D R$ in this conjugation experiment gives a good description of conjugation until the substrate is depleted. As a consequence of the low $\kappa_{c}$ value the model predicts conjugation to continue after the growth of the bacteria has reached stationary phase. By comparing the simulations of the donor and recipient concentrations Figure 3 with simulation of the transconjugant concentration 


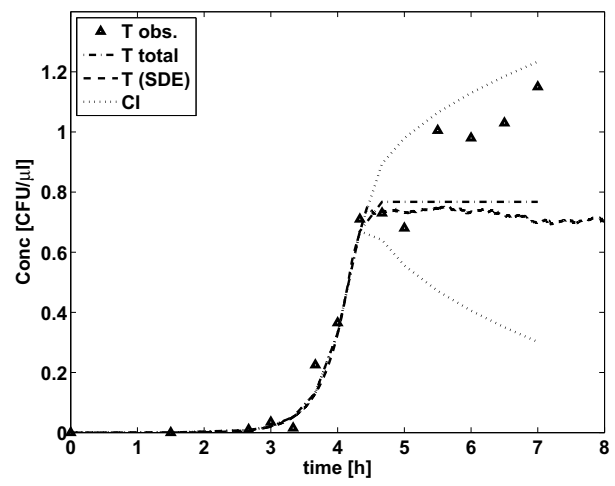

(a) Broth model

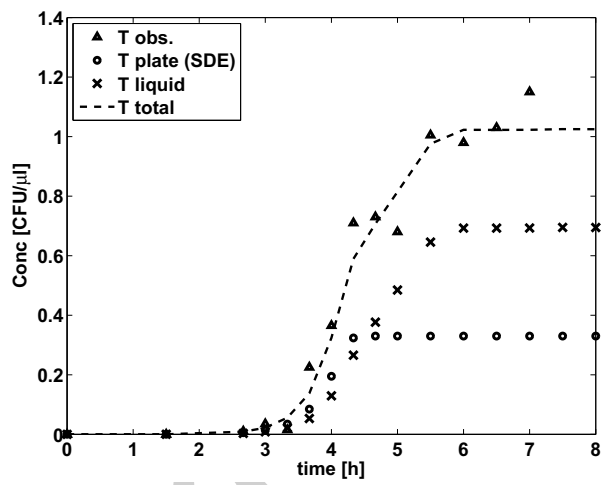

(b) Broth-plate model

Figure 4: The transconjugant concentration plotted together with simulations of the Broth and Broth-plate models. For the Broth model the mean of the simulated transconjugant concentration and its confidence interval, as estimated by CTSM, is shown. For each model one example of the SDE simulation made in Matlab is plotted.

Figure 4 we see that new transconjugants appear until approximately one hour after initiation of the stationary growth phase.

When calculating the bacterial concentration from the CFU count it was assumed that the data was Poisson distributed, and thus that the variance equals the mean. If this was indeed the case each of the parameters $\sigma_{4}^{2}, \sigma_{5}^{2}$, and $\sigma_{6}^{2}$ should equal one. In this case $\sigma_{4}^{2}$ is around 14 and $\sigma_{5}^{2}$ and $\sigma_{6}^{2}$ are around 
Table 2: The result of the ML parameter estimation for the Broth model and the Brothplate model.

\begin{tabular}{lllll}
\hline Parameter & \multicolumn{2}{l}{ Broth model $(\mathrm{SD})$} & $\begin{array}{l}\text { Broth-plate } \\
(\mathrm{SD})\end{array}$ & model \\
\hline$v_{D}\left[\mathrm{~h}^{-1}\right]$ & 1.678 & $(0.113)$ & 1.787 & $(0.092)$ \\
$v_{R}=v_{T}\left[\mathrm{~h}^{-1}\right]$ & 1.216 & $(0.028)$ & 1.261 & $(0.035)$ \\
$\kappa_{D}$ & 0.052 & $(0.047)$ & 0.110 & $(0.047)$ \\
$\kappa_{R}=\kappa_{T}$ & 0.0034 & $(0.0032)$ & 0.0364 & $(0.0199)$ \\
$\eta$ & $1.128 \cdot 10^{-6}$ & $\left(0.079 \cdot 10^{-6}\right)$ & $1.112 \cdot 10^{-6}$ & $\left(0.087 \cdot 10^{-6}\right)$ \\
$\kappa_{c}$ & $4.201 \cdot 10^{-16}$ & $\left(3.966 \cdot 10^{-16}\right)$ & $5.583 \cdot 10^{-15}$ & $\left(8.811 \cdot 10^{-15}\right)$ \\
$\gamma_{\max }\left[(\mathrm{CFU} / \mu \mathrm{l})^{-1} \mathrm{~h}^{-1}\right]$ & $4.913 \cdot 10^{-12}$ & $\left(0.636 \cdot 10^{-12}\right)$ & $2.008 \cdot 10^{-12}$ & $\left(0.699 \cdot 10^{-12}\right)$ \\
$\gamma_{p}^{\prime}\left[(\mathrm{CFU} / / \mu \mathrm{l})^{-1}\right]$ & 0 & - & $4.096 \cdot 10^{-12}$ & $\left(1.630 \cdot 10^{-12}\right)$ \\
$\sigma$ & $4.010 \cdot 10^{-10}$ & $\left(2.828 \cdot 10^{-10}\right)$ & $1.128 \cdot 10^{-11}$ & $\left(2.488 \cdot 10^{-11}\right)$ \\
$\sigma_{4}$ & 13.815 & $(5.349)$ & 14.862 & $(5.575)$ \\
$\sigma_{5}$ & 3.507 & $(1.212)$ & 3.572 & $(1.304)$ \\
$\sigma_{6}$ & 3.150 & $(1.422)$ & 3.184 & $(1.280)$ \\
\hline
\end{tabular}

3. This means that the data is over-dispersed, and this over-dispersion is accounted for by $\sigma_{4}^{2}, \sigma_{5}^{2}$, and $\sigma_{6}^{2}$. These parameters are in Poisson regression also refeered to as dispersion parameters. Several authors have found asymmetric likelihood profiles (Dennis et al., 2006; Ionides et al., 2006; Ponciano et al., 2007; King et al., 2008) in state-space models for dynamical biological systems (e.g. bird growth, plasmid persistence and cholera pandemic). In order to check the likelihood structure for our conjugation model, the profile-likelihood is calculated for $v_{D}$ and $\gamma_{\max }$. The profile likelihoods seen in Figure 5 are calculated by optimizing the likelihood function for fixed values of the parameter of interest. The $95 \%$ confidence interval (CI) plotted is the region of parameter values for which the profile log-likelihood value is larger than $\ell(\boldsymbol{\theta})_{\max }-c / 2$, where $\ell(\boldsymbol{\theta})_{\max }$ is the maximum log-likelihood value 
and $\mathrm{c}$ is defined by $\operatorname{Prob}\left[\chi^{2}(1)<c\right]=0.95$. The profile likelihoods are indeed asymmetric, which also in 3 out of 4 cases leads to asymmetric CI's. This is as expected due to the small number of observations. Furthermore it is seen that the profile likelihood CI is generally wider than the CI calculated by CTSM. However, this difference is not very large and we therefore believe that it has no or only a limited influence on the inference study performed. It should be noted that the observation noise seems to increase when the profile likelihood value is decreasing. This indicates that the observation noise (and not as expected the system noise) explains the difference between observations and the model. This is due to the few observations, which makes it difficult to adequately separate observation and system noise as discussed by Dennis et al. (2006).

In addition to the samples directly plated on the transconjugant plates, also 10 times concentrated samples were plated for the first six time points, where transconjugant concentration in the flask was low. However, an expected 100 times increase in conjugation on the plate compared to the non-concentrated sample was not observed. The reason for this is not clear. The sample is centrifuged in order to make a concentrated sample, this might change the ability for the donor and recipient to conjugate on the plate which could be one explanation. Further experiments should be performed to support the finding of conjugation under antibiotic pressure on the selective agar plate. 

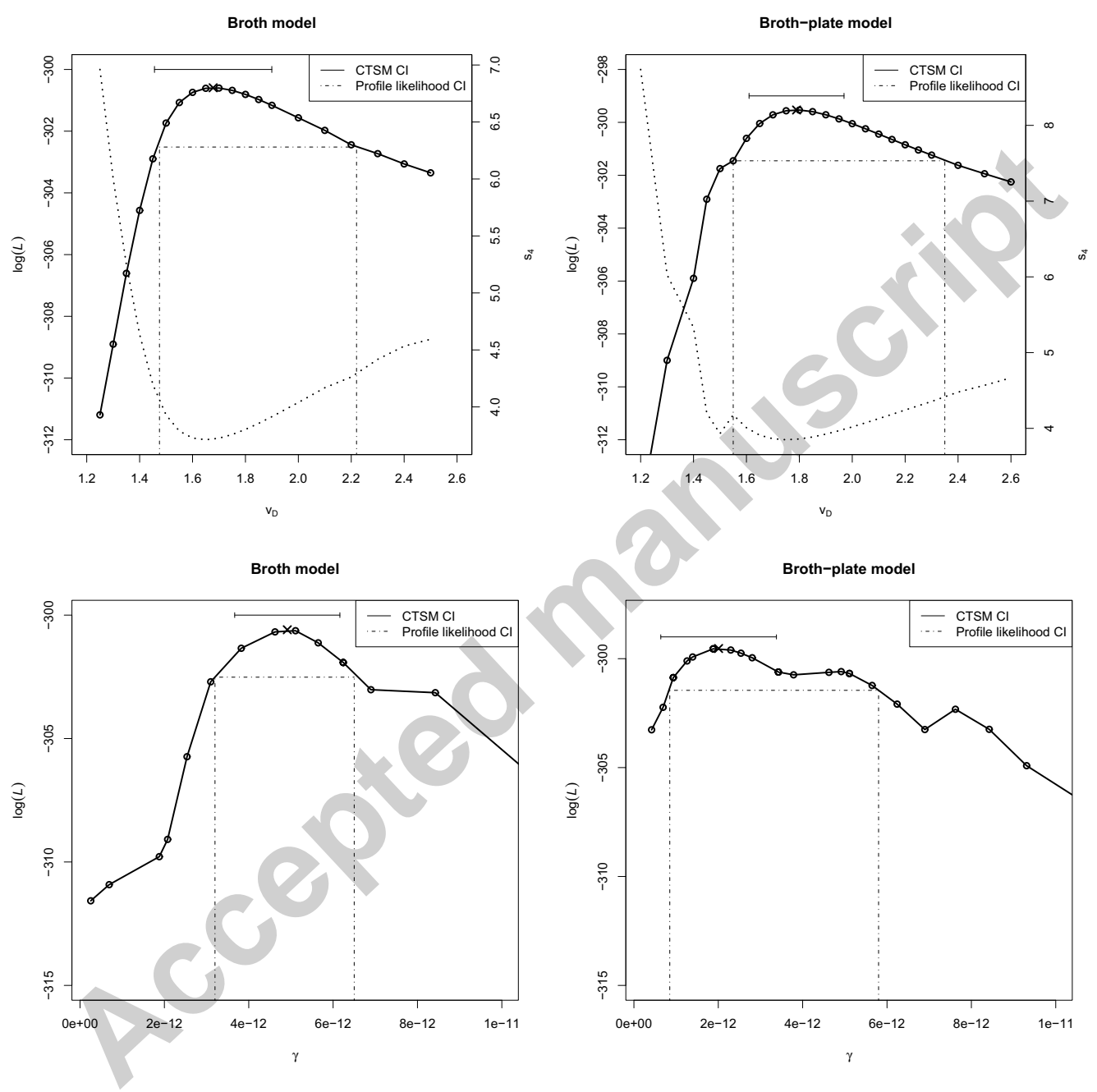

Figure 5: Profile likelihood (full line and circles) and approximate 95\% confidence interval for $v_{D}$ and $\gamma_{\max }$. The cross marks the estimated parameter value. The dotted lines in the top plots give the standard deviation $s_{4}$. 


\section{Conclusions}

The proposed SDE based state-space model is shown to successfully model conjugation in a broth exhaustible media. The suggested substrate dependent expression for the conjugation rate satisfactory model the conjugation process, which stops as the substrate is depleted. The ML based framework

for estimating model parameters combined with likelihood-ratio tests and AIC for inference studies provides strong tools for model improvements. It is shown that the stochasticity of the observations is best modelled as proportional noise.

The methodological problem of conjugation occurring on the transconjugant plates motivates the development of the Broth-plate model, which includes plate conjugation in the observation equation, whereby the plate conjugation can be separated from conjugation in the broth media. However, for the given data the Broth-plate model does not perform significantly better than the Broth-model.

\section{Acknowledgements}

This study was supported by the Danish Research Council for Technology and Production Sciences through the grant 274-05-0117 titled "Evolution and adaptation of antimicrobial resistance in bacterial populations". 
474

497

\section{A. Generalized linear model estimation}

For any given observation time $t_{k}$, the concentration $Y_{k}$ in the flask of a given bacteria population can be found from the following Matlab code:

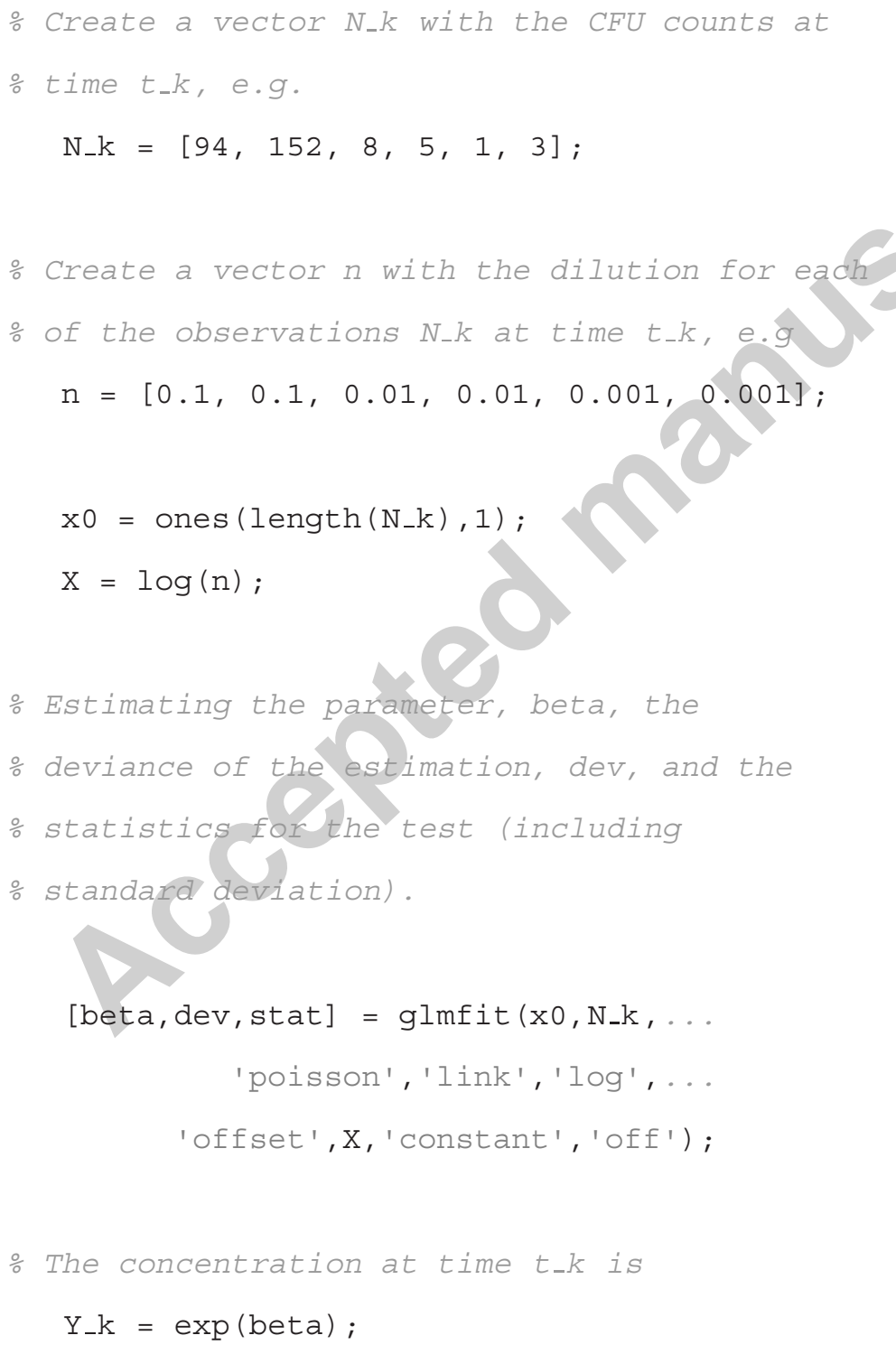


- The standard deviation at time t_k is sigma_Yk $=Y_{-} k *$ stat.se;

\section{References}

Andrup, L., Andersen, K., 1999. A comparison of the kinetics of plasmid transfer in the conjugation systems encoded by the f plasmid from Escherichia coli and plasmid pcf10 from Enterococcus faecalis. Microbiology 145 (8), 2001-2009.

Andrup, L., Smidt, L., Andersen, K., Boe, L., 1998. Kinetics of conjugative transfer: A study of the plasmid pxo16 from Bacillus thuringiensis subsp. israelensis. Plasmid 40 (1), 30-43.

Clewlow, L. J., Cresswell, N., Wellington, E. M. H., 1990. Mathematical model of plasmid transfer between strains of streptomycetes in soil microcosms. Appl. Environ. Microbiol. 56 (10), 3139-3145.

De Gelder, L., Ponciano, J. M., Abdo, Z., Joyce, P., Forney, L. J., Top, E. M., 2004. Combining mathematical models and statistical methods to understand and predict the dynamics of antibiotic-sensitive mutants in a population of resistant bacteria during experimental evolution. Genetics $168(3), 1131-1144$.

De Gelder, L., Ponciano, J. M., Joyce, P., Top, E. M., 2007. Stability of 
a promiscuous plasmid in different hosts: no guarantee for a long-term relationship. Microbiology (Reading, Engl.) 153 (Part 2), 452-463.

Dennis, B., Ponciano, J. M., Lele, S. R., 2006. Estimating density dependence, process noise, and observation error. Ecological Monographs 76 (3), 323.

Freter, R., Freter, R. R., Brickner, H., 1983. Experimental and mathematical models of Escherichia coli plasmid transfer in vitro and in vivo. Infect. Immun. 39 (1), 60-84.

Hasman, H., Aarestrup, F. M., 2002. Mechanisms of resistance - tcrB, a gene conferring transferable copper resistance in Enterococcus faecium: Occurrence, transferability, and linkage to macrolide and glycopeptide resistance. Antimicrob. Agents Chemother. 46 (5), 1410.

Higham, D. J., 2001. An algorithmic introduction to numerical simulation of stochastic differential equations. SIAM review 43 (3), 525-546.

Ionides, E. L., Bretó, C., King, A. A., 2006. Inference for nonlinear dynamical systems. Proc. Natl. Acad. Sci. U.S.A. 103 (49), 18438-18443.

Joyce, P., Abdo, Z., Ponciano, J. M., Gelder, L. D., Forney, L. J., Top, E. M., 2005. Modeling the impact of periodic bottlenecks, unidirectional mutation, and observational error in experimental evolution. J. Math. Biol. $50(6), 645-662$. 
King, A. A., Ionides, E. L., Pascual, M., Bouma, M. J., 2008. Inapparent infections and cholera dynamics. Nature 454 (7206), 877-880.

Klim, S., Mortensen, S. B., Kristensen, N. R., Overgaard, R. V., Madsen, H., 2009. Population stochastic modelling (psm) - an r package for mixedeffects models based on stochastic differential equations, computer methods and programs in biomedicine, in press. Comput. Methods Programs Biomed. 94 (3), 279-289.

Knudsen, G. R., Walter, M. V., Porteous, L. A., Prince, V. J., Armstrong, J. L., Seidler, R. J., 1988. Predictive model of conjugative plasmid transfer in the rhizosphere and phyllosphere. Appl. Environ. Microbiol. 54 (2), 343347.

Kristensen, N. R., Madsen, H., Jørgensen, S. B., 2004a. A method for systematic improvement of stochastic grey-box models. Computers and Chemical Engineering 28, 1431-1449.

Kristensen, N. R., Madsen, H., Jørgensen, S. B., 2004b. Parameter estimation in stochastic gray-box models. Automatica 40 (40), 225-237.

Levin, B., F.M., S., Rice, V., 1979. The kinetics of conjugative plasmid transmission: fit of a simple mass action model. Plasmid 2 (2), 247-260.

Levin, B. R., Stewart, F. M., 1980. The population biology of bacterial plasmids: A priori conditions for the existence of mobilizable nonconjugative factors. Genetics 94 (2), 425-443. 
MacDonald, Jacqueline, A., Smets, Barth, F., Rittmann, Bruce, E., 1992. Effects of energy availability on the conjugative-transfer kinetics of plasmid rp4. Water Res. 26 (4), 461-468.

Monod, J., 1949. The growth of bacterial cultures. Annu. Rev. Microbiol. 3, 371-394.

Ponciano, J. M., Gelder, L. D., Top, E. M., Joyce, P., 2007. The population biology of bacterial plasmids: A hidden markov model approach. Genetics $176(2), 957$.

Slater, F. R., Bailey, M. J., Tett, A. J., Turner, S. L., 2008. Progress towards understanding the fate of plasmids in bacterial communities. FEMS Microbiol. Ecol. 66 (1), 3-13.

Sudarshana, P., Knudsen, G. R., 2006. Quantification and modeling of plasmid mobilization on seeds and roots. Curr. Microbiol. 52 (6), 455-459.

Tier, C., Floyd, H., 1981. Persistence in denisty dependent stochastic populations. Math. Biosci. 53, 89-117.

Top, E., Vanrolleghem, P., Mergeay, M., Verstraete, W., 1992. Determination of the mechanism of retrotransfer by mechanistic mathematical modeling. J. Bacteriol. 174 (18), 5953-5960.

Willms, A., Roughan, P., Heinemann, J., 2006. Static recipient cells as reser- 
${ }_{581}$ voirs of antibiotic resistance during antibiotic therapy. Theor. Popul. Biol. $58270(4), 436-451$. 